\title{
Disseminated aspergillosis
}

\author{
B. J . B URKE, F. K. STORR I N G, a nd T. E. P A R R Y \\ Assisted Respiration Unit and Department of Clinical Pathology, Llandough Hospital \\ (United Cardiff Hospitals), Penarth, Glamorgan
}

\begin{abstract}
The history and the clinical and necropsy findings are presented of a patient who died from Aspergillus fumigatus pyaemia occurring as an opportunistic infection complicating a dissecting aortic aneurysm. The diagnosis was made (and treatment instituted) during life: it rested upon repeated isolation of the organism from tracheal aspirations, a positive blood culture and positive serum precipitin reactions to $A$. fumigatus. Debilitating disease along with large doses of antibiotics and corticosteroids provided the conditions necessary for the normally saphrophytic fungi to become pathogenic. As the therapy for aspergillosis is still ineffective, the danger of prescribing large doses of antibiotics together with corticosteroids is stressed.
\end{abstract}

Interest in the fungal diseases and especially those caused by the aspergillus species has greatly increased over the past decade. As a result, attempts to culture aspergilli are being made more frequently and the advent of skin and precipitin tests has enabled the distinction to be made between contamination and infection. Even so it is extremely rare for the diagnosis of disseminated aspergillosis to be made during life (Caplan, Frisch, Houghton, Climo, and Natsios, 1968 ; Rifkind, Marchioro, Schneck, and Hill, 1967); the diagnosis is usually made by histological and cultural examination of necropsy specimens. We present a case of disseminated aspergillosis diagnosed during life and treated accordingly. The gross debility of the patient and the therapeutic barrage to which he was subjected provided the conditions for a disseminated fungal infection.

\section{CASE REPORT}

A 51-year-old man employed as a store-keeper in a car factory was admitted to hospital because of severe throbbing pain between the scapulae and also in the lumbar region. Thirteen years earlier the upper and middle lobes of the right lung were resected for caseating tuberculosis. For two years before admission he had been treated for severe fluctuating systemic hypertension.

On examination he was in severe pain and there was some tenderness and guarding in the right hypochondrium. All the pulses were present and strong; the blood pressure was $240 / 100 \mathrm{~mm}$. Hg. Later, on the day of admission, he developed a grey facies and profuse sweating. A leaking abdominal aortic aneurysm was diagnosed and a laparotomy was performed on the following day. At laparotomy the findings were those of a slight aneurysmal dilatation of the aorta just above the aortic bifurcation with some oedema of the adventitia. Free blood was not present in the abdomen. No surgical procedure was undertaken and the abdomen was closed. On the fourth post-operative day he complained of lumbar pain and was noted to be slightly jaundiced; serum bilirubin was $6.9 \mathrm{mg} . / 100 \mathrm{ml}$. and the haemoglobin 13.9 g. $/ 100 \mathrm{ml}$. The blood pressure was $260 / 160 \mathrm{~mm}$. $\mathrm{Hg}$, and the temperature varied between $35.5^{\circ} \mathrm{C}$. and $37 \cdot 5^{\circ}$ C. A septicaemia was suspected and after three blood cultures had been taken cephaloridine was given intravenously (blood cultures proved negative). On the sixth post-operative day he suddenly became profoundly shocked and breathless. He was intubated and intermittent positive pressure ventilation was started. The chest radiograph was suggestive of a leftsided pleural effusion, but repeated aspiration produced $2 \mathrm{ml}$. of fresh blood only. In view of his condition hydrocortisone, $1.2 \mathrm{~g} . / 24$ hours, and also chloramphenicol, cloxacillin, and ampicillin were given intravenously. On the next day his haemoglobin had fallen to $8.5 \mathrm{~g} . / 100 \mathrm{ml}$., so he was transfused with $2,800 \mathrm{ml}$. blood. An aortogram was considered, but he was too ill to be moved.

On the eleventh post-operative day the hydrocortisone was discontinued, the dose having been slowly reduced over the previous fow days. A profuse growth of $A$. fumigatus and a scanty growth of Candida albicans were obtained from the tracheal aspirate. A heavy growth of $A$. fumigatus was obtained from tracheal aspirates on three further occasions during the next seven days. The organism was identified by the slide culture method of Riddell (1951) (Fig. 1). C. albicans was grown from two of these as well as from one oesophageal aspirate and from two swabs taken from the tongue during the same period. On the thirteenth post-operative day he had a pyrexia of $39^{\circ} \mathrm{C}$. and complained of colicky back pain. A chest radiograph showed that the changes in the left side of the thorax were resolving 


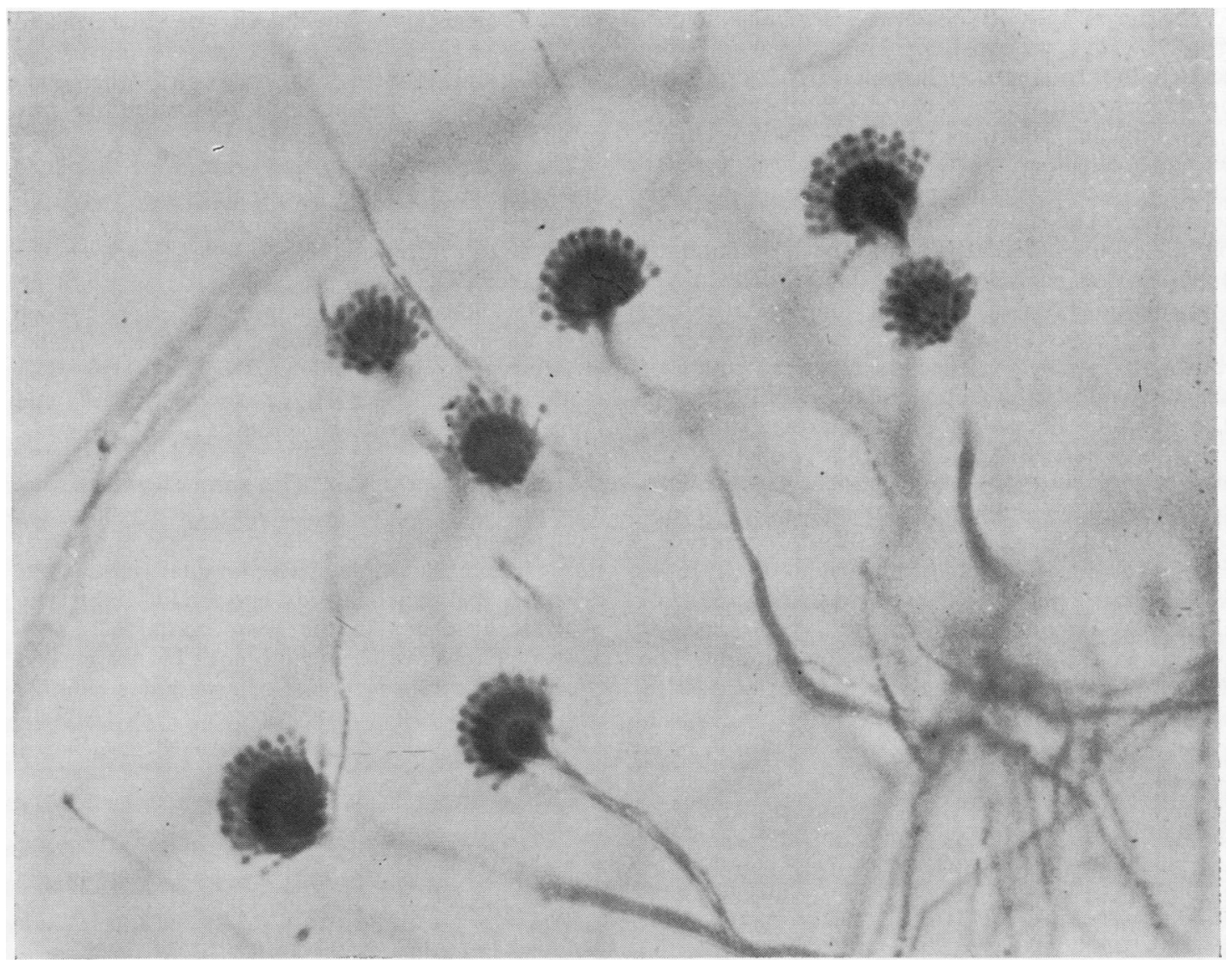

FIG. 1. Slide culture of A. fumigatus showing conidial heads. $\times 800$.

but that there were signs of consolidation in the right lung. Because of his deteriorating condition hydrocortisone, 1 g. $/ 24$ hours i.v., was recommenced, along with carbenicillin and cloxacillin after three more blood cultures had been taken (sterile after 10 days' incubation). There was no blood or sputum eosinophilia and the haemoglobin was now $12 \mathrm{~g} . /$ $100 \mathrm{ml}$. Two days later his haemoglobin had dropped to $9 \mathrm{~g} . / 100 \mathrm{ml}$. and he was transfused with $1,100 \mathrm{ml}$. of blood. Intradermal skin testing to Aspergillus 1\% antigen (Bencard Moulds group $M$ 2) and to A. fumigatus $1 \%$ antigen (Bencard) gave neither an immediate type I nor a delayed type III response. Serum precipitins against $A$. fumigatus were tested in two different laboratories. One reported a strong, and the other a moderately strong, positive reaction.

On the fourteenth post-operative day natamycin (Pimaracin) inhalations were begun in a dosage of $2.5 \mathrm{mg}$. 8-hourly nebulized via the Bennett ventilator. Two days later he became unconscious; a blood culture taken in Sabouraud's agar medium then grew two colonies of $A$. fumigatus. The following day a lumbar puncture was performed ; C.S.F. pressure was increased to $250 \mathrm{~mm}$. The fluid contained 4 red and 4 white cells $/ \mathrm{cu}$. $\mathrm{mm}$. On routine culture the C.S.F. was sterile. From this time on he remained unconscious and despite treatment with amphotericin B intravenously for three days he died 24 days after admission.

NECROPSY FINDINGS A dissecting aortic aneurysm was found as the underlying disease. The aorta showed severe atheromatous change particularly in the descending thoracic and abdominal parts. The dissecting aneurysm took its origin from a tear in the posterior wall of the aortic arch and extended along the descending thoracic and abdominal aorta, re-entering the latter shortly above the aortic bifurcation. An extensive haematoma was present around the descending thoracic aorta with a leakage into the left pleural cavity. 
The heart showed bilateral ventricular hypertrophy. Tiny yellowish pyaemic abscesses were scattered throughout the anterior, lateral and posterior walls of the left ventricle.

The trachea and the larger bronchi showed extensive sloughing of the lining mucosa and the bronchial tree contained abundant purulent secretion. The right pleural cavity was obliterated by adhesions; the left pleural cavity contained $1,200 \mathrm{ml}$. of partially clotted blood. The upper and middle lobes of the right lung were absent. The right lower lobe was congested and oedematous and revealed on section scattered areas of haemorrhagic bronchopneumonic consolidation. The left lung likewise showed extensive bronchopneumonic consolidation, particularly of the lower lobe, with haemorrhagic appearances in parts. Evidence of active pulmonary tuberculosis was not found, but two small foci of calcification were noted in the subapical region of the right lower lobe.

The gastric mucosa showed tiny scattered erosions. A large area of acute ulceration was present in the first part of the duodenum. The liver presented the nutmeg appearances of passive venous congestion. The spleen was enlarged and soft.

The kidneys were congested and when sectioned small recent infarcts and multiple tiny pyaemic abscesses were seen.

The convolutions of the brain were flattened and on section extensive softening was found, involving both cerebral hemispheres, the basal ganglia and almost the entire right cerebellar hemisphere.

Bacteriological examination of swabs taken from the right lung at necropsy yielded Monilia, $A$. fumigatus and Escherichia coli. Swabs taken from the brain grew $A$. fumigatus, $E$. coli and Bacterium faecalis alkaligenes.

HISTOLOGICAL FINDINGS The aorta showed severe atheromatous lesions extending deeply into the media with medial necrosis and extensive destruction of the elastic tissue. The luminal walls of the dissecting channel were endothelialized. The pyaemic abscesses in the heart contained abundant hyphae, showing dichotomous branching and transverse septation typical of aspergillus (Fig. 2).

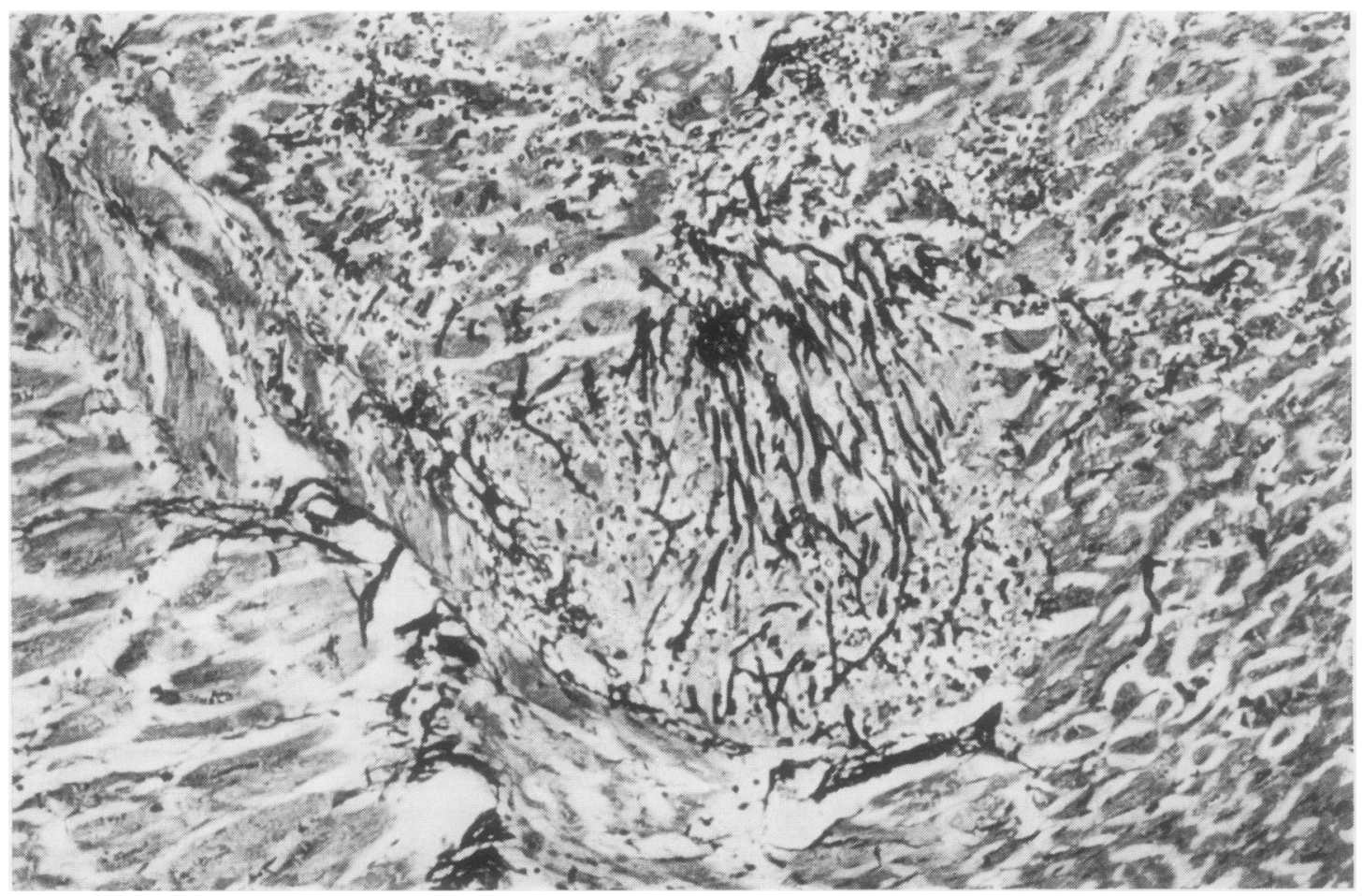

FIG. 2. Myocardium. Pyaemic abscess with abundant hyphae showing dichotomous branching and frequent septa. Methenamine silver nitrate. $\times 250$. 
The epithelial lining of the trachea had sloughed off and was replaced by a thick layer of fungus growth. Conidial heads were seen amongst the abundant septate and lateral branching hyphae (Fig. 3). Similar features were seen in the large bronchi with invasion of the tracheal and bronchial walls by hyphae (Fig. 4).

Sections taken from various parts of the lungs showed widespread bronchopneumonic lesions and numerous vascular thrombi causing multiple small infarcts in which fungal growth was particularly abundant. Hyphae were also noted in several blood vessels (Fig. 5).

Sections of the acute duodenal ulcer showed infarction of the mucosa with abundant surface proliferation of budding yeast cells and septate mycelia. The hyphae were relatively small and did not show dichotomous branching and were thus identified as those of Candida.

Sections of the liver showed focal centrilobular necrosis. No hyphae were seen in either the liver

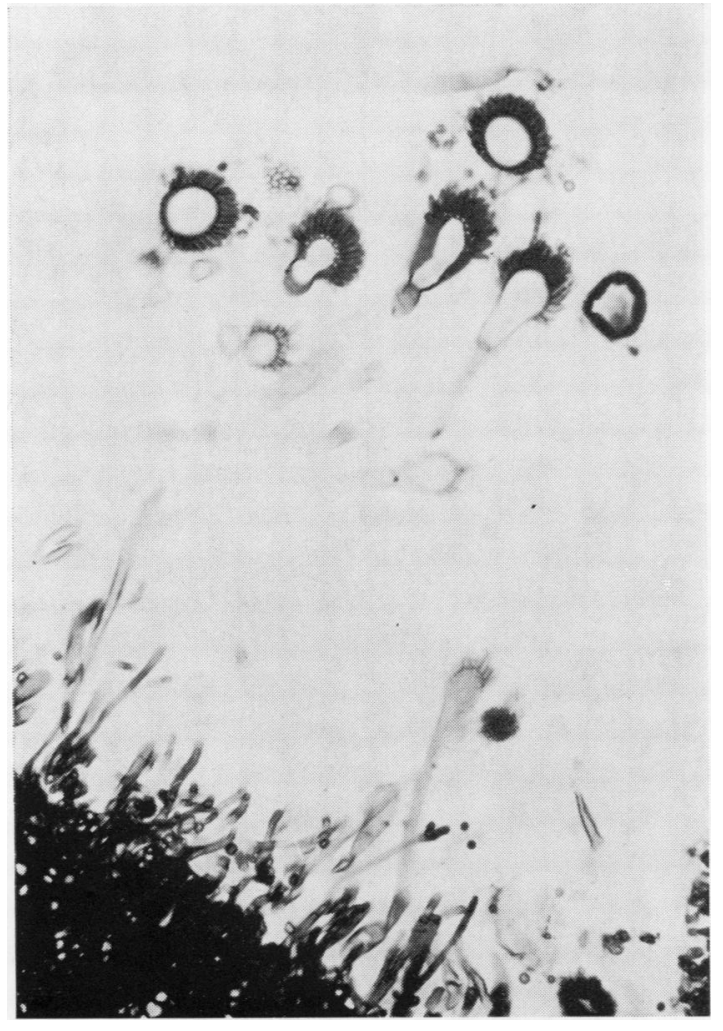

FIG. 3. Trachea coated with a thick layer of fungal growth at bottom left with almost complete conidial heads at top. Methenamine silver nitrate. $\times 800$.

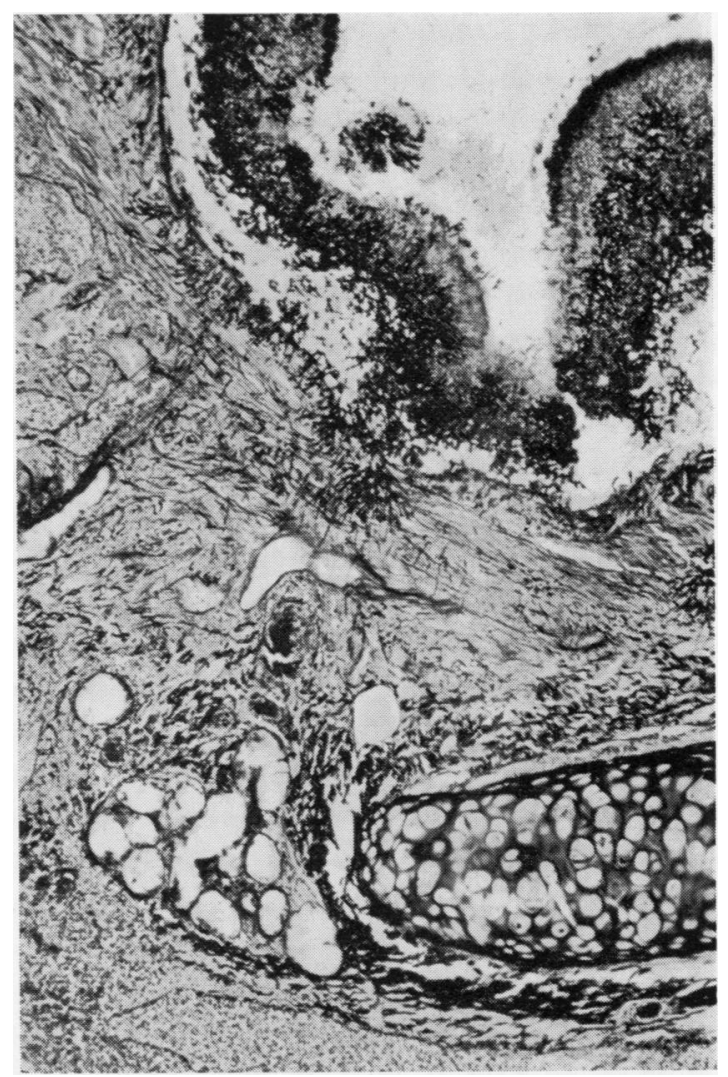

FIG. 4. Bronchial wall coated with, and invaded by, aspergilli. Methenamine silver nitrate. $\times 250$.

or the spleen. Abundant septate and branching hyphae were present in the pyaemic abscesses and in the infarcts of both kidneys.

Sections taken from various parts of the brain showed extensive cerebral and cerebellar softening with widespread vascular thromboses and perivascular haemorrhages. Abundant hyphae with the histological characteristics of aspergillus were present within vessels and were seen to invade the brain tissue (Fig. 6).

The cause of death was considered to be $A$. fumigatus septicaemia complicating a leaking dissecting aortic aneurysm.

\section{DISCUSSION}

It is extremely rare to obtain a positive blood culture of $\boldsymbol{A}$ fumigatus. Blood cultures have been negative even when taken, by trans-septal puncture with a cardiac catheter, from the left atrium of a 


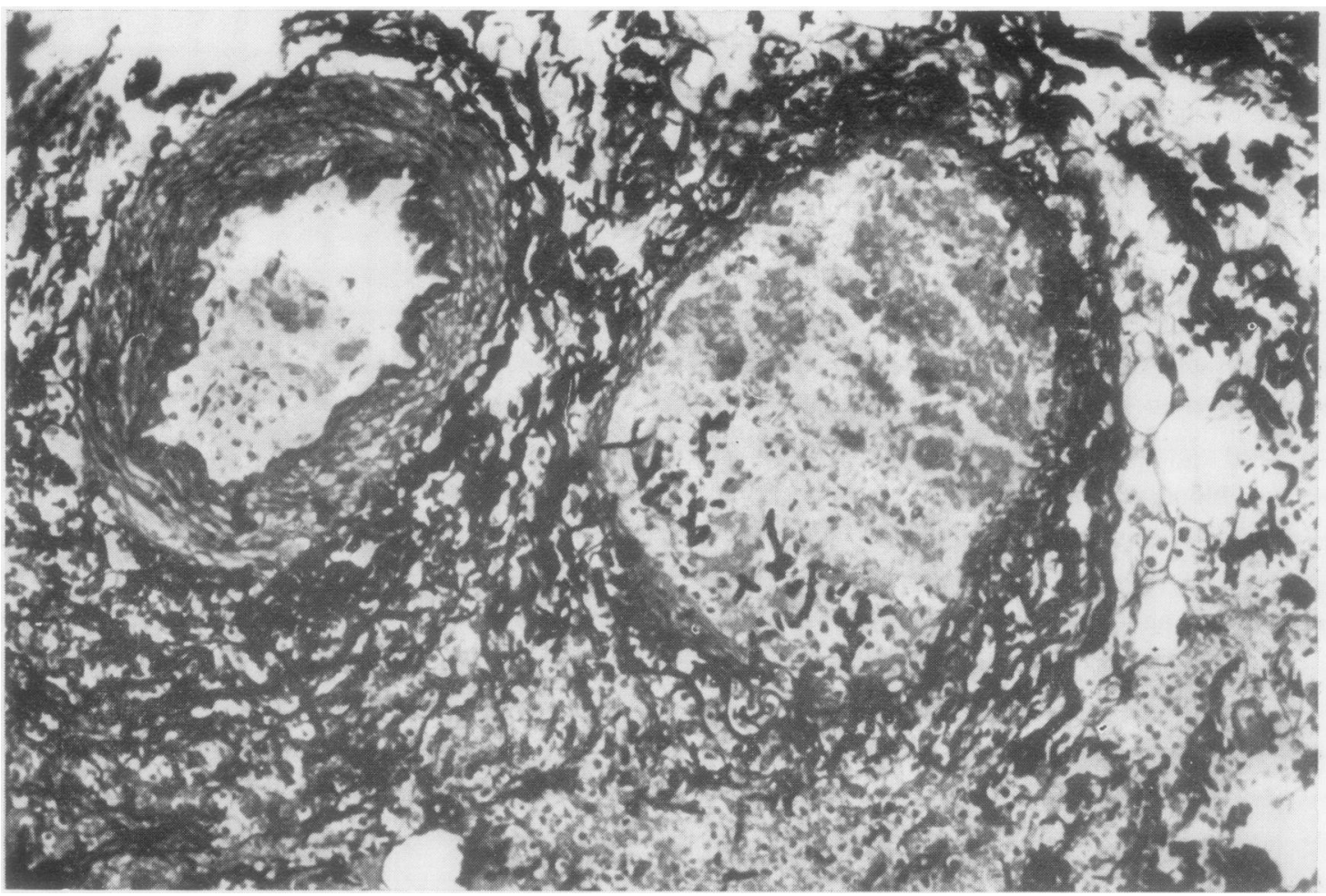

FIG. 5. Lung. Aspergilli invading a thrombosed vein. Methenamine silver nitrate. $\times 250$.

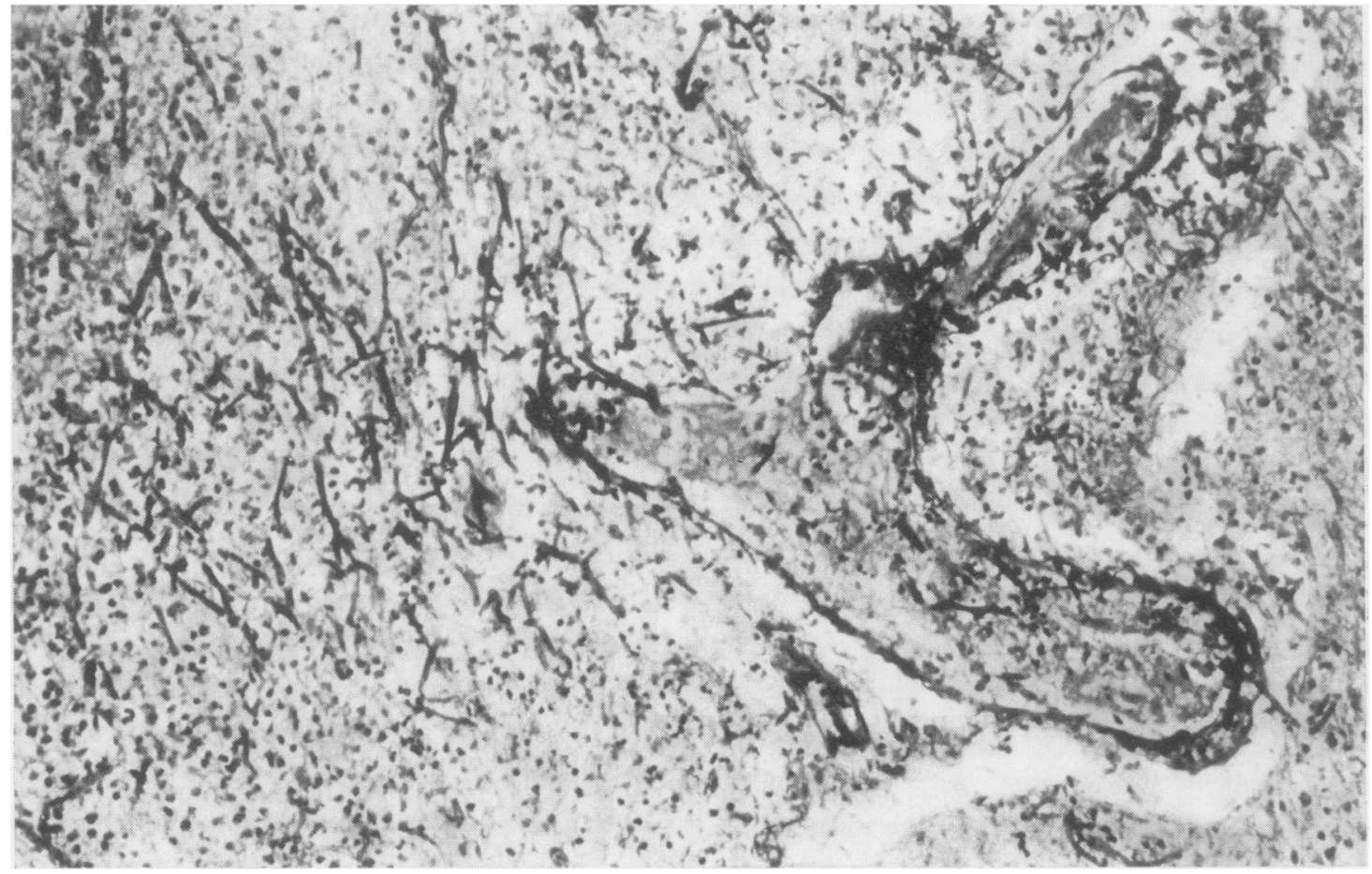

FIG. 6. Brain. Thrombosed vessel and area of softening, both invaded by aspergilli. Methenamine silver nitrate. $\times 250$. 
patient proved at necropsy to have prosthetic valve endocarditis due to $A$. fumigatus (Dr. R. M. E. Seal, personal communication of a case to be published). Our methods consisted of liquefying solid Sabouraud's medium by heating and then allowing it almost to solidify before adding $5 \mathrm{ml}$. of blood obtained aseptically by antecubital venesection. The mixture was then poured onto a plastic Petri dish and incubated at $37^{\circ} \mathrm{C}$. As this was done by the patient's bedside, aerial contamination of the plate cannot be completely excluded; this, however, is unlikely in view of the short exposure and because the patient was on a ventilator with an expiratory air filter.

Thirteen confirmed cases of disseminated aspergillosis were described in the literature before 1960 (Grcevic and Matthews, 1959). The numbers increased and Khoo, Singai, and Leong (1966), in describing their case, brought the total up to 23. In none of these was a diagnosis possible before death.

As aspergilli are commonly present as saprophytes in the respiratory tract their culture from the sputum does not necessarily indicate aspergillosis. This term should be reserved for cases in which the presence of aspergilli has led to an inflammatory or hypersensitivity response or where the fungi have become invasive.

Treatment of non-invasive bronchopulmonary aspergillosis has been discussed by Henderson and Pearson (1968). Amphotericin B (Henderson, English, and Vecht, 1968 ; Caplan et al., 1968) is the current treatment for disseminated aspergillosis but it is only fungistatic and slowly acting so that a 30-day course is required. In view of the fulminating nature of the disease, once it has become invasive, treatment with amphotericin B is unlikely to be successful. Aspergilli usually enter the blood stream by invasion of thrombosed pulmonary veins (Grcevic and Matthews, 1959). Thus the finding of aspergilli in the sputum, although by no means proof of an invasive lesion, should always be taken seriously, especially if the patient is receiving antibiotics as well as corticosteroids.

Neither an immediate (type I) nor a late (type III) skin reaction to aspergillus antigen is proof of active infection (Pepys, Riddell, Citron, Clayton, and Short, 1959 ; Scadding, 1967), but the demonstration of serum precipitins to aspergilli almost always indicates the presence of live fungus in the body (Avila, 1968). As these disappear relatively quickly when contact with the infecting fungus has ceased, their presence indicates an active or recent fungus infection (Henderson, 1968 ; Henderson, et al., 1968). They were present in only $1 \%$ of the sera of 1,000 urban blood donors (R. M. E. Seal, personal communication). High titres are obtained in patients with invasive aspergillosis and moderately high titres in $60 \%$ of patients with the asthmatic type of aspergillosis and also in $90 \%$ of patients with aspergilloma (Henderson et al., 1968).

In the present case repeated heavy growths of A. fumigatus from the tracheal aspirates, a positive blood culture and a positive serum precipitin reaction enabled the diagnosis of disseminated aspergillosis to be made during life, which was confirmed by histology and cultures at necropsy.

We thank Mr. Hugh Jones, Dr. J. Donald Ball, and Dr. A. J. Thomas for permission to publish the details of this patient who was under their care, and Dr. H. R. Dingle, Dr. R. M. E. Seal, and Dr. D. A. Williams for helpful criticism of the manuscript. We are grateful to Dr. Mary English and Dr. R. M. E. Seal for performing the serum precipitin tests and to Miss S. E. Perry for technical assistance.

\section{REFERENCES}

Avila, R. (1968). Immunological study of pulmonary aspergilloma Thorax, 23, 144.

Caplan, H. I., Frisch, E., Houghton, J. D., Climo, M. S., and Natsios, G. A. (1968). Aspergillus fumigatus endocarditis. Ann. intern. Med., 68, 378.

Grcevic, M., and Matthews, W. F. (1959). Pathologic changes in acute disseminated aspergillosis. Amer. J. clin. Path., 32, 536.

Henderson, A. H. (1968). Allergic aspergillosis: review of 32 cases. Thorax, 23, 501.

English, M. P., and Vecht, R. J. (1968). Pulmonary aspergillosis Thorax, 23, 513 .

and Pearson, J. E. G. (1968). Treatment of bronchopulmonary aspergillosis with observations on the use of natamycin. Thorax, $23,519$.

Khoo, T. K., Sugai, K., and Leong, T. K. (1966). Disseminated aspergillosis. Amer. J. clin. Path., 45, 697.

Pepys, J., Riddell, R. W., Citron, K. M., Clayton, Y. M., and Short, E. I. (1959). Clinical and immunological significance of aspergillus fumigatus in the sputum. Amer. Rev. resp. Dis., 80, 167.

Riddell, R. W. (1951). Laboratory diagnosis of common fungus infections. In Recent Advances in Clinical Pathology, 2nd ed. edited by Dyke, S. C., p. 77. Churchill, London.

Rifkind, D., Marchioro, T., Schneck, S., and Hill, R. B. (1967). Systemic fungal infections complicating renal transplantation, and immunosuppressive therapy. Amer. J. Med., 43, 28.

Scadding, J. G. (1967). Antigen-antibody reactions in bronchopulmonary disease. J. roy. Coll. Phycns Lond., 2, 35. 